\title{
Study of Water Cherenkov muon detector parameters and performances for LHAASO
}

\section{Xiurong Li}

Key Laboratory of Particle Astrophysics, Institute of High Energy Physics, CAS, Beijing 100049, China. E-mail: lixr@ihep.ac.cn

Huihai He*

Key Laboratory of Particle Astrophysics, Institute of High Energy Physics, CAS, Beijing 100049, China. E-mail: hhh@ihep.ac.cn

\section{Gang Xiao}

Key Laboratory of Particle Astrophysics, Institute of High Energy Physics, CAS, Beijing, China.

\section{Xiong Zuo}

Key Laboratory of Particle Astrophysics, Institute of High Energy Physics, CAS, Beijing, China.

\section{Shaohui Feng}

Key Laboratory of Particle Astrophysics, Institute of High Energy Physics, CAS, Beijing, China.

\section{Cong Li}

Key Laboratory of Particle Astrophysics, Institute of High Energy Physics, CAS, Beijing, China.

FOR THE LHAASO COLLABORATION

\begin{abstract}
In the Large High Altitude Air Shower Observatory project, about 1146 water Cherenkov muon detectors will be built to discriminate cosmic ray species. Each muon detector has a $6.8 \mathrm{~m}$ diameter Tyvek bag containing $1.2 \mathrm{~m}$ height pure water in it. In the summer of 2014, a water Cherenkov muon detectors prototype was installed at YBJ, both data analysis and simulation has been performed. The detector performances strongly depend on detector parameters, especially the Tyvek reflectivity and water attenuation length. MD prototype signals are simulated by using Geant 4 with different parameters, while the detector parameters are determined by comparing simulation to data. The MD performances, such as NPE and the relationship of effective attenuation length with water absorption length, Tyvek reflectivity and time ranges are studied.
\end{abstract}

The 34th International Cosmic Ray Conference,

30 July- 6 August, 2015

The Hague, The Netherlands

\footnotetext{
* Speaker.
} 


\section{Introduction}

The Large High Altitude Air Shower Observatory(LHAASO) is designed to search for cosmicray sources by the measurement of gamma rays and to study cosmic-ray physics from $10 \mathrm{TeV}$ to a few hundreds of PeVs [1]. It consists of $1 \mathrm{~km}^{2}$ array (KM2A), 90,000 $\mathrm{m}^{2}$ water Cherenkov detector array (WCDA), 24 wide-filed Cherenkov telescopes(WFCTA), and $5000 \mathrm{~m}^{2}$ shower core detector array(SCDA). As a main array of LHAASO, KM2A has two major targets, i.e. searching sources of ultrahigh energy cosmic ray and studying cosmic ray physics in an energy range from $20 \mathrm{TeV}$ to $100 \mathrm{PeV}$ and is expected to reach a sensitivity of $1 \% \mathrm{Crab}$ flux unit for $\gamma$-ray point sources at $50 \mathrm{TeV}$, which requires getting at least $10 \gamma$ rays from a single source each year. To measure such a low flux, a detector array with an effective area of one square kilometer working with full duty cycle to reach background-free detection of gamma rays at this energy is needed. Measuring the numbers of electromagnetic components and muons in an EAS can highly discriminate primary gamma rays from the cosmic nuclei because gamma showers are muon-poor. Through detailed Monte Carlo simulations [2][3] and different designs of the KM2A array are studied. The final design for LHAASO-KM2A is one square kilometer with 5242 electromagnetic particle detectors (EDs) and 1146 muon detectors (MDs). The EDs provide the main useful information on the injected primary cosmic rays or gamma rays, such as energy and direction, while shower muons are detected by muon detectors.

For LHAASO-KM2A, each MD includes a $6.8 \mathrm{~m}$ diameter and $1.2 \mathrm{~m}$ height concrete tank containing a sealed liner with a reflective inner Tyvek surface and 44t of pure water. Cherenkov photons produced by the passage of particles through the water is collected by one eight-inchdiameter PMT which is in the top center of the linear that looks downwards. Dirt above a MD will be used to shield against the shower electromagnetic components. [4]. In the summer of 2014, a water-Cherenkov muon detector prototype same with the designed MD detectors was installed at YBJ (4300m asl). Right of Fig. 1 is the schematic view of the MD prototype. The detailed data performances of the MD prototype can be found at papers [5] [6]. Signals of background single muon with random zenith angles are measured with average number of photoelectron (NPE) to be about 72.5 and the average attenuation time to be about $200 \mathrm{~ns}$. MD prototype signals are simulated by using Geant4 [7] with different parameters, while the detector parameters are determined by comparing simulation to data. The MD performances, such as NPE and the relationship of effective attenuation length with water absorption length, Tyvek reflectivity and time ranges are studied.

\section{The Geant4 simulation of MD prototype}

When a charged particle with high speed passes through the MD water, it generates per centimeter about 300 Cherenkov photons with wavelength in 300-600 nm and Cherenkov angles about $42^{\circ}$. The energy threshold to generate Cherenkov photons in water is about $0.77 \mathrm{MeV}$ for electrons and $160 \mathrm{MeV}$ for muons. The number of photons generated is similarly proportional to $1 / \lambda^{2}$ and $E^{2}$ of the photons(the wave length $\lambda$ and energy of photon $E$ has the relation: $E \times \lambda=1240 \mathrm{eV}$ ). Some of the photons can be absorbed by water or the Tyvek, and some can hit the PMT to generate signals. After each reflection by the liner, a photon has a chance probability of about $5 \times 10^{-4}$ to hit the PMT photocathode. The total number of photons in the water and also the number of 

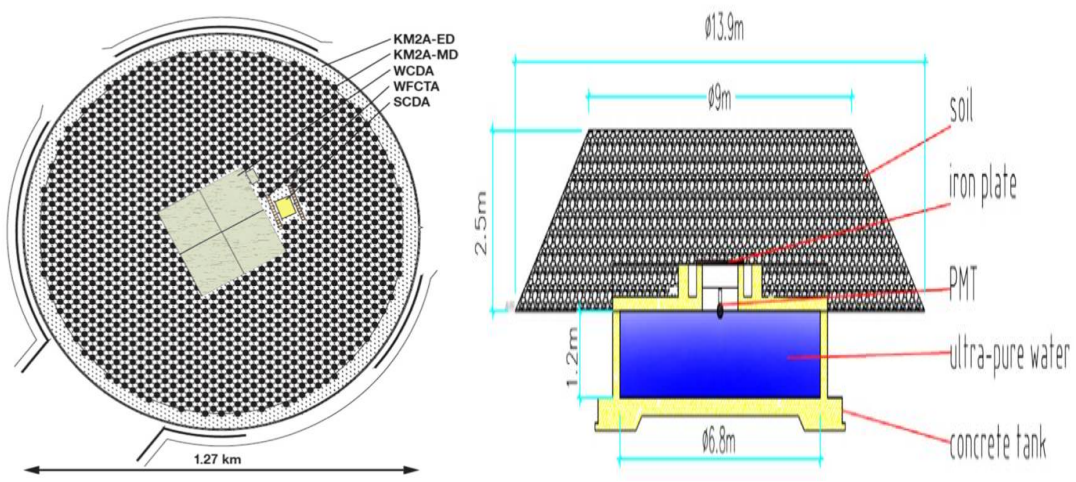

Figure 1: Left: The overview of LHAASO array; Right: The schematic view of the MD prototype

photons hitting the PMT decreases approximately exponentially with time and distance, and the decrease factor is mainly decided by water absorption length, Tyvek reflectivity and geometry of the detector. For the same water, water absorption length $\lambda$ and Tyvek reflectivity $f$ varies with the wavelength of photons, reaching the maximum values at about $400 \mathrm{~nm}$. The maximum absorbing length and reflectivity depends on the water purity and the Tyvek performance.

The geometry of the prototype in simulation is same with the real detector, and the energy of muons is set as $3 \mathrm{GeV}$. The azimuth angles are set randomly between 0 to $2 \pi$, and $\cos ^{3}$ (zenith) is selected from uniform random numbers between 0 and 1 . For the depending factor of water absorbing length and Tyvek reflectivity on wave length of photons, we use the same values that used in Auger water tank simulation [8]. We do simulation with different maximum absorbing length $\lambda_{\max }$ and maximum reflectivity $f_{\max }$, and compare the results between data and simulation to get the detector parameters. Left of Fig. 2 shows the simulated average NPE of single muon with different $\lambda_{\max }$ and $f_{\max }$, while right of Fig. 2 is NPE distribution from simulation of single muons with $\lambda_{\max } 200 \mathrm{~m}$ and $f_{\max } 98.5 \%$. We fit the time distribution of photons collected by the PMT in the time range from $50 \mathrm{~ns}$ to $650 \mathrm{~ns}$ with a exponentially function to get the effective attenuation time. Left of Fig. 3 is the fitted effective attenuation time from simulation with different $\lambda_{\max }$ and $f_{\max }$, right of Fig. 3 is time distribution of photons hitting PMT from simulation of thousands of single muons with $\lambda_{\max } 200 \mathrm{~m}$ and $f_{\max } 98.5 \%$. Cherenkov photons have different wave length with different absorbing length and Tyvek reflectivity, so the PMT time distribution is the sum of different exponential functions and can not be fitted well with one standard exponential function in the whole range. The simulation with $\lambda_{\max } 200 \mathrm{~m}$ and $f_{\max } 98.5 \%$ can get similar signal results with real data, so we think the simulation using maximum Tyvek reflectivity $98.5 \%$ and $\lambda_{\max } 200$ $m$ can approximatively describe the MD prototype.

\section{The attenuation length}

When $N_{0}$ residual photons with certain absorbing length $\lambda$ and reflectivity $f$ propagate in the water for the distance $\mathrm{x}$, the number of photons becomes $N_{0} \times e^{-\frac{x}{\lambda}} \times f^{\frac{x}{L}}$. The mean times of reflection is $\frac{x}{L}$, where the mean track length of photon between two bounces with liner (step length L) can be got from simulation. Left of Fig. 4 shows the averaged photon step length versus number 

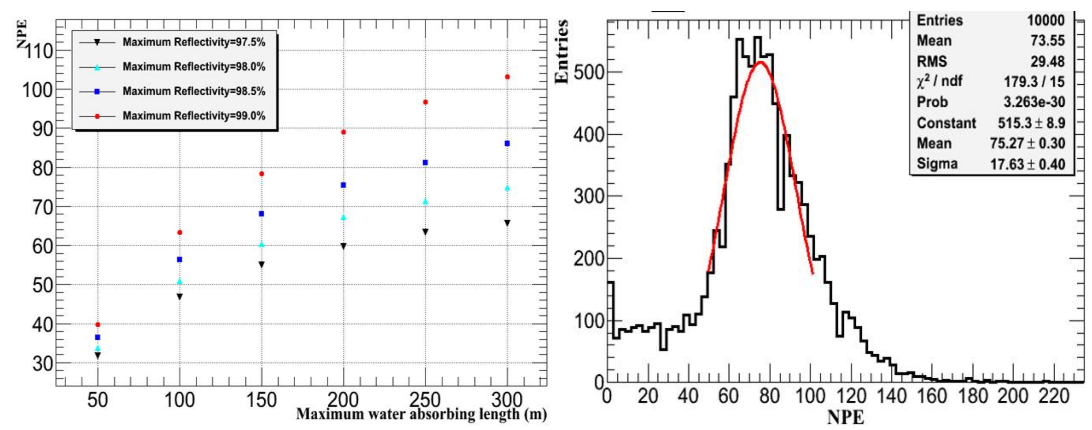

Figure 2: Left:Averaged NPE from simulation of single muons with different water absorbing length and Tyvek reflectivity; Right:NPE distribution from simulation of single muons with maximum water absorbing length $200 \mathrm{~m}$ and maximum Tyvek reflectivity $98.5 \%$
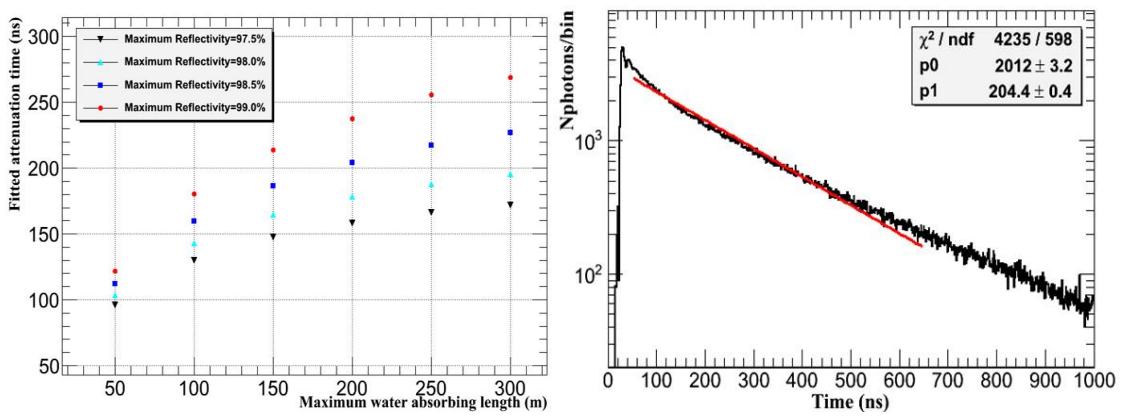

Figure 3: Left:Fitted effective attenuation time from simulation of single muons with different maximum water absorbing length and Tyvek reflectivity; Right: Time distribution of photons hitting PMT from simulation of single muons with maximum water absorbing length $200 \mathrm{~m}$ and Tyvek reflectivity $98.5 \%$

of reflection from the MD prototype simulation, and right is the averaged photon step length versus PMT time. We can see from simulation that the mean step length becomes stable about $1.8 \mathrm{~m}$ after 30 times of reflection. Photons decrease with distance exponentially with the effective attenuation length $\lambda_{e}$, which is decided by the combined effect of $\lambda$ and $f$ with the relationship:

$$
e^{-\frac{x}{\lambda_{e}}}=e^{-\frac{x}{\lambda}} \times f^{\frac{x}{L}}
$$

The above formula can be changed to the form:

$$
\frac{1}{\lambda_{e}}=\left(\frac{1}{\lambda}-\frac{\ln f}{L}\right)
$$

The PMT waveform is the amplitude of photons at different time, which is the response of the number of photons in the tank at different time. The PMT area is small enough compared to the MD surface area and the absorbing of PMT can be ignored. The attenuation time $\tau$ of the waveform can be got by fitting the smooth part of the PMT waveform, as well as the effective attenuation length $\lambda_{e}$ which is $\tau \times \frac{c}{n}$. If the photons in the tank has the fixed water attenuation length $\lambda$ and Tyvek reflectivity $f$, the PMT waveform and number of photons will attenuate with the formula $e^{-t \times \frac{c}{n} \times\left(\frac{1}{\lambda}-\frac{\ln f}{L}\right)}$, where $\mathrm{c}$ is the light velocity in vacuum and $\mathrm{n}$ is the refractive index of water.

In average for the muon signals, the number of the first bunch of photoelectrons collected by the PMT is about 5, which is dependent on the impinging position and the direction of a muon 
track. The routes of photons become random after several times of reflection, and the PMT waveform becomes smooth meanwhile. The wavelength of Cherenkov photons spreads from $300 \mathrm{~nm}$ to $600 \mathrm{~nm}$, and photons with different wavelength have different water absorbing length and Tyvek reflectivity. At first the number of Cherenkov photons generated is similarly proportional to the square of photon energy. When the photons propagate in the tank, the photons with small absorbing length and reflectivity are easily to be absorbed firstly and the photons with bigger absorbing length and reflectivity can last longer time. The decrease factor of total number of photons in the water and also the number of photons hitting the PMT should depend on the photon time, where the initial time is the trigger time of the muon event. When we fit the PMT waveform at different time range, the effective attenuation length should becomes bigger when the fit range becomes latter.

We have studied the depending effect of effective absorbing length on time in detail by simulation. We get the averaged photon energy at each different $100 \mathrm{~ns}$ range of PMT time and found the energy changes with time, which is showed in left of Fig. 5. Then the averaged absorbing length $\lambda$ and reflectivity $f$ at different time range can be got based on the depending factor of Tyvek reflectivity and absorbing length on photon energy at different time. The averaged $f$ got at different time range is showed in right of Fig. 5 and averaged $\lambda$ is showed in left of Fig. 6. We use formula(3.2) to calculate the effective attenuation length with the $\lambda$ and $f$ got at different time range, which is showed by the black points in the right of Fig. 6 . At the same time, we fit the simulated PMT waveform to get the fitted effective attenuation length $\lambda_{e}$, which is showed by the red marks in the right of Fig. 6. The effective absorbing length $\lambda_{e}$ calculated out and fitted out is similar, and becomes bigger when the fit range is latter. From simulation we can see that the effective attenuation length depends on time and becomes bigger with time, which can be calculated out from formula(3.2) and fitted out with the PMT waveform. The performance for the real data is similar but a little more complicated because a jitter in the signal trigger time makes the averaged waveform more flatter.
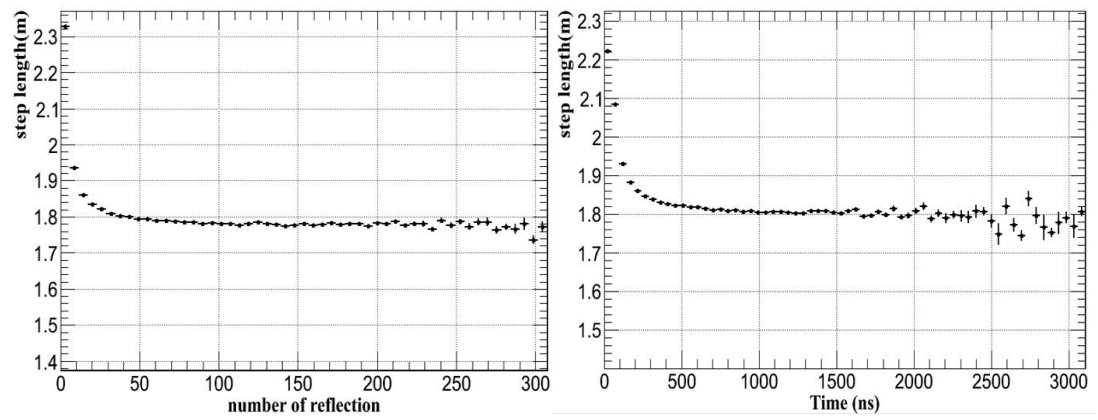

Figure 4: Left: The averaged photon step length versus number of reflection from the MD prototype simulation; Right: The averaged photon step length versus PMT time.

\section{Summary and Conclusions}

A muon detector prototype was constructed at YBJ cosmic ray observatory in the summer of 2014 and both data analysis and simulation has been done. The parameters of the MD prototype are got, and the NPE and waveform of data and simulation is in good consistence. The relationship of effective attenuation length with water absorbing length, Tyvek reflectivity, photon step length 

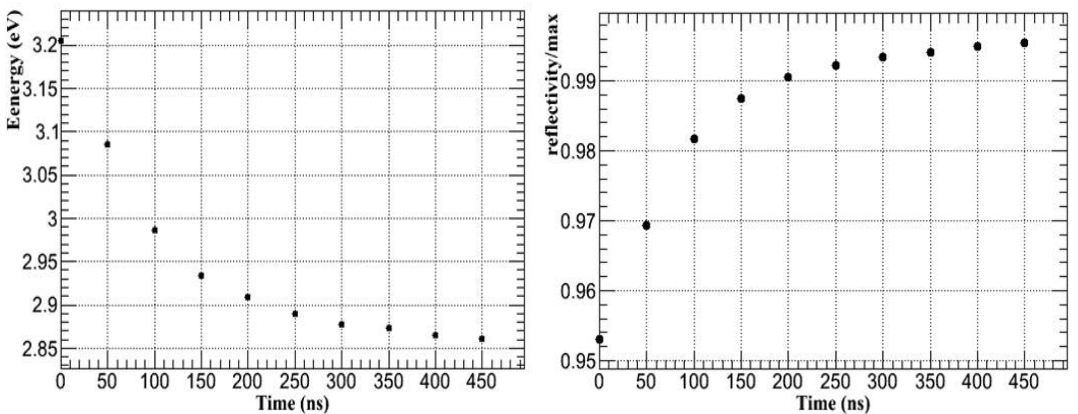

Figure 5: Left: The averaged photon energy at different time range; Right:The averaged reflectivity $f$ at different time range
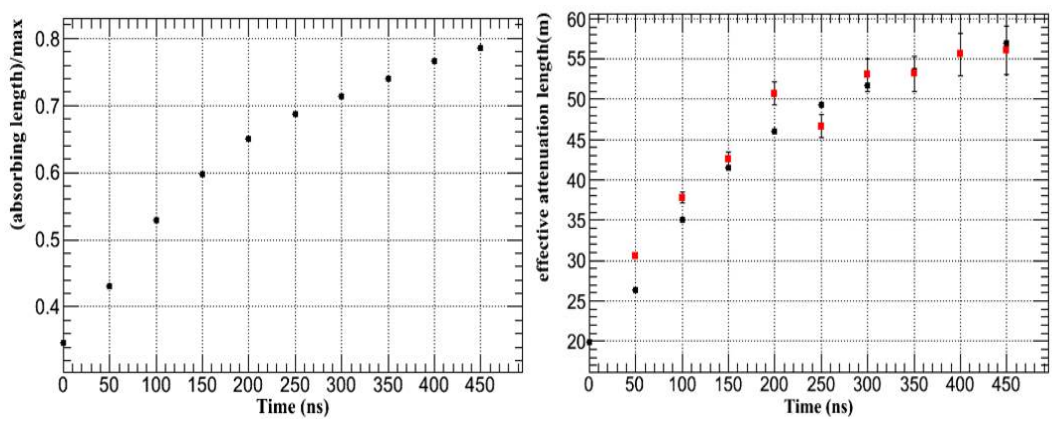

Figure 6: Left: The averaged absorbing length $\lambda$ at different time range; Right:The effective attenuation length $\lambda_{e}$ at different time range

and time range is studied in detail. The effective absorbing length fitted out from PMT waveform changes with the time range, which is found to be consistent with the values decided by water absorbing length, Tyvek reflectivity and photon step length.

Acknowledgement: This work is supported in China by NSFC (NO.11375210,No.11375224, No.11405181, No.11475190), the Chinese Academy of Science, Institute of High Energy Physics, the Key Laboratory of Particle Astrophysics, CAS.

\section{References}

[1] Z. Cao et al, LHAASO Collaboration, in:Proceedings of 31st ICRC, 2009.

[2] X.H. Ma et al., LHAASO Collaboration, in: Proceedings of 31st ICRC, 2009.

[3] S.W. Cui et al., LHAASO Collaboration, in: Proceedings of 32st ICRC, 2011.

[4] G. X et al., LHAASO Collaboration, in: Proceedings of 33st ICRC, 2011.

[5] X. Z et al., Nuclear Instruments and Methods in Physics Research A 789 (2015) 143

[6] X. Z., Posdoctoral thesis of Chinese academic of science, 2015

[7] J. Allison, et al., IEEE Transactions on Nuclear Science NS-53(1)(2006)270.

[8] P. L. Ghia et al., PIERRE AUGER Collaboration, in: Proceedings of 30st ICRC, 2007. 